\title{
Investigating the effect of porosity level and pore former type on the mechanical and corrosion resistance properties of agro-waste shaped porous alumina ceramics
}

\begin{abstract}
The strength integrity and chemical stability of porous alumina ceramics operating under extreme service conditions are of major importance in understanding their service behavior if they are to stand the test of time. In the present study, the effect of porosity and different pore former type on the mechanical strength and corrosion resistance properties of porous alumina ceramics have been studied. Given the potential of agricultural wastes as pore-forming agents (PFAs), a series of porous alumina ceramics (A12O3-xPFA; $\mathrm{x}=5,10,15$ and $20 \mathrm{wt} \%$ ) were successfully prepared from rice husk $(\mathrm{RH})$ and sugarcane bagasse (SCB) through the powder metallurgy technique. Experimental results showed that the porosity (44-67\%) and the pore size $(70-178 \mu \mathrm{m})$ of porous alumina samples maintained a linear relationship with the PFA loading. Comprehensive mechanical strength characterization of the porous alumina samples was conducted not just as a function of porosity but also as a function of the different PFA type used. Overall, the mechanical properties showed an inverse relationship with the porosity as the developed porous alumina samples exhibited tensile and compressive strengths of 20.4-1.5 MPa and 179.5-10.9 MPa respectively. Moreover, higher strengths were observed in the SCB shaped samples up to the $15 \mathrm{wt} \%$ PFA mark, while beyond this point, the silica peak observed in the XRD pattern of the RH shaped samples favored their relatively high strength. The corrosion resistance characterization of the porous alumina samples in hot $10 \mathrm{wt} \% \mathrm{NaOH}$ and $20 \mathrm{wt} \% \mathrm{H} 2 \mathrm{SO} 4$ solutions was also investigated by considering sample formulations with 5-15 wt\% PFA addition. With increasing porosity, the mass loss range in $\mathrm{RH}$ and SCB shaped samples after corrosion in $\mathrm{NaOH}$ solution for $8 \mathrm{~h}$ were $1.25-3.6 \%$ and $0.44-2.9 \%$ respectively; on the other hand, after corrosion in $\mathrm{H} 2 \mathrm{SO} 4$ solution for $8 \mathrm{~h}$, the mass loss range in RH and SCB shaped samples were $0.62-1.5 \%$ and $0.68-3.3 \%$ respectively.
\end{abstract}

Keyword: Porous alumina; Agro-waste PFA; Porosity; Mechanical properties; Corrosion resistance 